\title{
3. Länger zufrieden arbeiten? Qualität und Ausgestaltung von Erwerbstätigkeit in der zweiten Lebenshälfte
}

\author{
Janna Franke \& Martin Wetzel
}

\section{Kernaussagen}

Im Jahr 2014 sind mehr Menschen in der Lebensphase vor dem Ruhestand erwerbstätig als 1996: Im Jahr 2014 waren 74,1 Prozent der 40- bis 65-Jährigen erwerbstätig, während es 1996 noch 60,2 Prozent waren. Die Anteile der Erwerbstätigen im Alter von 54 bis 59 Jahren sowie von 60 bis 65 Jahren haben sich von 1996 zu 2014 sogar um je 20 Prozentpunkte erhöht (1996: 56,6 Prozent, 2014: 76,1 Prozent für 54- bis 59-Jährige; 1996: 18,2 Prozent, 2014: 38,8 Prozent für 60- bis 65-Jährige). Der Anteil der erwerbstätigen Personen im Alter von 60 bis 65 Jahren liegt jedoch auch 2014 deutlich unter dem Durchschnitt der erwerbstätigen Personen im Alter von 40 bis 65 Jahren (74,1 Prozent).

Belastungen im Rahmen der Erwerbstätigkeit unterscheiden sich zwischen den Bildungsgruppen: Fast die Hälfte (47,4 Prozent) aller Erwerbstätigen im Alter von 40 bis 65 Jahren berichtet im Jahr 2014 zeitliche und nervliche Belastungen. Knapp ein Drittel (31,2 Prozent) der Erwerbstätigen berichtet von körperlichen Belastungen bei der eigenen Tätigkeit. Während zeitliche und nervliche Belastungen vor allem von Personen mit einem hohen Bildungsniveau berichtet werden, werden körperliche Belastungen bei der Arbeit eher von Personen mit einem niedrigen Bildungsniveau angegeben.

Im Jahr 2014 berichten mehr Erwerbstätige von Belastungen als 2002: Zeitliche und nervliche Belastungen haben zwischen 2002 und 2014 von 43,3 Prozent auf 47,4 Prozent zugenommen. Körperliche Belastungen haben im gleichen Zeitraum von etwa einem Viertel (25,3 Prozent) auf ein knappes Drittel (31,2 Prozent) zugenommen.

Der Großteil der Erwerbstätigen ist im Jahr 2014 zufrieden mit ihrer Arbeit und fühlt sich weder über- noch unterfordert: Mehr als vier Fünftel (85,2 Prozent) der Erwerbstätigen sind im Jahr 2014 zufrieden mit ihrer eigenen Arbeit. Nur 2,8 Prozent fühlen sich über- und 16,1 Prozent unterfordert. Der Großteil der Erwerbstätigen empfindet ihre Arbeitsanforderungen als genau richtig (81,2 Prozent).

Ein zunehmender Anteil an Menschen ist auch nach dem Eintritt in den Ruhestand erwerbstätig: Der Anteil der Erwerbstätigen im Ruhestand nimmt von 5,1 Prozent im Jahr 1996 auf 11,6 Prozent im Jahr 2014 zu. Erwerbstätig sind im Jahr 2014 vor allem Männer zwischen 60 und 71 Jahren sowie Personen mit einem hohen Bildungsniveau. 


\subsection{Einleitung}

Der Arbeitsmarkt wird derzeit nachhaltig durch den demografischen Wandel verändert. So steht eine abnehmende Anzahl von Erwerbstätigen einer größer werdenden Anzahl an Personen im Ruhestand gegenüber (Büsch, Dittrich, \& Lieberum 2010). Daraus erwachsen neue Herausforderungen sowohl für das Erwerbssystem (zum Beispiel Fachkräftemangel) als auch für das Rentensystem (zum Beispiel Finanzierbarkeit). Diesen aktuellen Herausforderungen versucht die Politik zum einen mit einer schrittweisen Erhöhung des gesetzlichen Renteneintrittsalters und zum anderen durch die Verringerung von vorzeitigen Ruhestandsregelungen $\mathrm{zu}$ begegnen (Brussig 2009). Auch wenn die

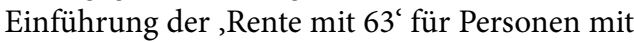
45 Beitragsjahren einen vorzeitigen Eintritt in den Ruhestand ermöglicht ${ }^{1}$, zielt die derzeitige Arbeitsmarktpolitik auf eine längere Integration älterer Erwerbstätiger in den Arbeitsmarkt ab. In diesem Kapitel soll daher untersucht werden, was diejenigen Personen ausmacht, die eine längere Erwerbsbeteiligung sowohl vor als auch im Ruhestand aufweisen und mit welchen Herausforderungen diese Personen in der Arbeitswelt konfrontiert sind.

Aktuelle Studien zeigen, dass ältere Erwerbspersonen häufig länger im Berufsleben verbleiben. Eine steigende Erwerbsbeteiligung konnte in den letzten Jahren vor allem im Alter vor dem offiziellen Ruhestandseintritt beobachtet werden (Bundesagentur für Arbeit 2013; vgl. dazu Kapitel 4). Es zeigen sich jedoch auch deutliche Unterschiede zwischen Personengruppen. So ist die Erwerbsbeteiligung älterer Menschen sehr unterschiedlich in den Niveaus und Trends nach

1 Da die Rente mit 63 erst seit dem 01. Juli 2014 bezogen werden kann, sollte der Einfluss dieser Reform auf die Erwerbstätigenzahlen der DEAS-2014-Befragung recht gering sein. Für die zukünftigen Jahre ist zu vermuten, dass die Einführung der Rente mit 63 zu einem leichten Aufhalten des allgemeinen Prozesses der längeren Erwerbsbeteiligung führt, aber den allgemeinen Prozess aufgrund ihres besonderen Zuschnittes auf Personen mit hochstabilen Erwerbsbiografien und kurzen Ausbildungszeiten nicht deutlich verändert.
Geschlecht, Bildung und Region verteilt. Forschungsergebnisse weisen darauf hin, dass Männer immer noch häufiger im Alter zwischen 60 und 64 Jahren erwerbstätig sind als Frauen, auch wenn der Anteil berufstätiger Frauen in dieser Altersgruppe wächst (Mümken \& Brussig 2012). Weiterhin wurde gezeigt, dass über 75 Prozent der Personen mit Hochschulabschluss im Alter von 55 bis 64 Jahren noch erwerbstätig sind, während dies in der gleichen Altersgruppe der Personen ohne Berufsabschluss auf weniger als 40 Prozent zutrifft (Asef \& Wingerter 2011; Brussig 2010). Zwischen Ost- und Westdeutschland hat sich die Erwerbsbeteiligung in späteren Lebensphasen in den letzten beiden Jahrzehnten angeglichen, jedoch sind die regionalen Unterschiede (vor allem für Frauen) noch deutlich zu erkennen (Asef \& Wingerter 2011).

Durch die aktuelle Erhöhung der gesetzlichen Regelaltersgrenze verschieben sich die Erwerbsteilnahmechancen älterer Erwerbspersonen. Vor allem Alters- und Bildungsunterschiede könnten einen Einfluss auf den Erwerbsaustrittszeitpunkt haben (vgl. Kapitel 4). In diesem Kapitel soll beleuchtet werden, wo Handlungsbedarf zur Verbesserung der Arbeitsmarktintegration und der Arbeitsbedingungen besteht. Gibt es die sogenannten älteren Erwerbstätigen? Oder wählen gerade Personen mit niedrigem Bildungsniveau besonders häufig einen vorzeitigen Ruhestandseintritt aufgrund von hohen Belastungen? Wenn ja, dann würde ein nach Bildung unterschiedlicher Austritt auch ungleiche Einbußen bei der finanziellen Sicherung im Alter nach sich ziehen (vgl. Kapitel 4).

Die Möglichkeiten einer verlängerten Erwerbstätigkeit werden jedoch nicht nur von gesetzlichen und gesundheitlichen Faktoren (vgl. Kapitel 8) beeinflusst. Für den Verbleib in der Erwerbstätigkeit spielen neben der Arbeitszufriedenheit auch Belastungssituationen im Erwerbsalltag sowie Über- und Unterforderung durch die Arbeit eine wichtige Rolle (Bäcker, Brussig, Jansen, Knuth, \& Nordhause-Janz 2009). Nachteilige Arbeitsbedingungen können $\mathrm{zu}$ gesundheitlichen Beeinträchtigungen, Fehl- 
zeiten oder Arbeitsunfähigkeit und dadurch zum vorzeitigen Austritt aus dem Erwerbsleben führen (Bödeker, Friedel, Friedrichs, \& Röttger 2008; Wurm 2004). Zudem beeinflussen (Un-) Zufriedenheit und Belastungen im Erwerbsleben auch die Wahrscheinlichkeit, im Ruhestand weiterhin erwerbstätig zu sein (Büsch et al. 2010). Um ein besseres Bild möglicher Einflussfaktoren auf die Erwerbstätigkeit älterer Arbeitnehmer zu erhalten, werden in diesem Kapitel zusätzlich zu den Erwerbsquoten vor und nach dem Ruhestandsübergang Arbeitszufriedenheit, Arbeitsbelastungen durch zeitliche und nervliche sowie körperliche Belastungen, ebenso wie die Bewertung der Passung zwischen Arbeitsanforderung und eigener Fähigkeit in den Blick genommen.

Bisherige Forschungsergebnisse deuten darauf hin, dass die Zufriedenheit mit der eigenen Arbeit von einem Großteil der Erwerbstätigen als hoch bewertet wird (von Rosenstiel 2009; Wurm 2004). Dabei wurden keine signifikanten Unterschiede zwischen Männern und Frauen beobachtet. Uneinheitlicher ist die Befundlage, wenn die Arbeitszufriedenheit nach Bildung differenziert betrachtet wird. Einige Studien argumentieren, dass die Tätigkeiten von Personen mit hohem Bildungsniveau häufiger anregende und fordernde Inhalte aufweisen als die Tätigkeiten von Personen mit niedrigem Bildungsniveau und daher Bildung positiv mit Arbeitszufriedenheit zusammenhängt (von Rosenstiel 2009). Andere Studien zeigen jedoch, dass weder die Arbeitsbedingungen noch sozio-ökonomische Merkmale die Arbeitszufriedenheit bestimmen, sondern primär Persönlichkeitsmerkmale (Brenke 2015).

Belastungssituationen im Erwerbsleben können durch verschiedene Faktoren ausgelöst werden. Häufig wird zwischen gesundheitlichen Belastungen und Stress unterschieden. Stress ist die Arbeitsbelastung, unter der Erwerbstätige am häufigsten leiden und die in den letzten Jahren am stärksten zugenommen hat (Wurm 2004; Bäcker et al. 2009). Von Stress als Belastung berichten vor allem Männer mit hohem Bildungsniveau (Wurm 2004). Auch körperliche Belastungen scheinen zuzunehmen (Bäcker et al. 2009). Das ist insofern bemerkenswert, da durch die fortschreitende Technisierung ein Rückgang körperlicher Belastung zu vermuten wäre. Eine mögliche Erklärung ist, dass auch Bürotätigkeiten als einseitige körperliche Belastungen wahrgenommen werden können. Besonders, langes Sitzen' wird als Belastung empfunden. Trotzdem berichten Personen mit hohem Bildungsniveau durchschnittlich seltener körperlich belastet $\mathrm{zu}$ sein als Personen mit niedrigem Bildungsniveau (Bäcker et al. 2009).

Die subjektive Bewertung der Arbeitsbelastung wird zudem wesentlich durch die Passung zwischen individueller Leistungsfähigkeit und Anforderungen der Tätigkeit beeinflusst. Stimmen die Arbeitsanforderungen und die Fähigkeiten der Erwerbstätigen nicht überein, kann es zu Unter- beziehungsweise Überforderung kommen. Ein häufig geäußertes Vorurteil geht von einer Abnahme der Leistungsfähigkeit älterer Erwerbstätiger aus (Bal, Reiss, Rudolph, \& Baltes 2011; vgl. dazu Kapitel 22), wodurch vermeintlich für ältere Erwerbstätige eine steigende Überforderung trotz konstantem Anforderungsprofil der Tätigkeit entstehen würde. Einer solchen Abnahme der Leistungsfähigkeit mit dem Alter widersprechen jedoch bisherige Studien (Ng \& Feldman 2008; Lohmann-Haislah 2012; Behrend 2002; von Rosenstiel 2009). Diese im Berufsalltag verbreiteten Vorurteile zur Leistungsfähigkeit Älterer könnten aber dazu führen, dass ältere Erwerbstätige stärker einseitige Tätigkeiten ausüben sollen und sich dadurch unterfordert fühlen (Behrend 2002).

Das vorliegende Kapitel legt den Fokus darauf, wie sich die Erwerbspartizipation und Arbeitsbedingungen älterer Erwerbstätiger über die Zeit entwickelt haben. Dafür werden zuerst die Erwerbsquoten für verschiedene Personengruppen über die Zeit betrachtet. Daraufhin wird die Erwerbssituation von Personen unterschiedlicher Alters- und Bildungsniveaus in Bezug auf die Arbeitszufriedenheit, Belastung und Passung berücksichtigt.

Nicht nur die Erwerbspartizipation vor dem Ruhestand steigt, auch Personen im Ruhestand sind immer häufiger erwerbstätig. Im Jahr 2008 arbeiteten überwiegend westdeutsche Männer, Personen mit einem hohen Bildungsabschluss und Selbstständige nach dem Übergang in den 
Ruhestand (Brussig 2010; Engstler \& Romeu Gordo 2014). Ein Anliegen dieses Kapitels ist es zu untersuchen, ob sich dieser Trend im Allgemeinen und für verschiedene Personengruppen weiter fortgesetzt hat.

Im vorliegenden Kapitel werden die folgenden Fragen untersucht:

1. Wie hat sich die Erwerbsbeteiligung älterer Erwerbspersonen seit 1996 verändert?
2. Wie bewerten verschiedene Gruppen von Erwerbstätigen ihre Arbeit? Wie haben sich diese Indikatoren über die Zeit verändert?

3. Hat die Erwerbsbeteiligung im Ruhestand seit 2008 weiter zugenommen? Wer arbeitet im Ruhestand und wie wird Erwerbstätigkeit im Ruhestand gestaltet?

fasst: „Wie zufrieden sind Sie derzeit mit Ihrer Arbeitssituation insgesamt?" Für die Analysen wird nur zwischen den Zufriedenen (,sehr zufrieden'/,eher zufrieden') und den Nichtzufriedenen (,teils, teils',eher unzufrieden'/,sehr unzufrieden') unterschieden.

Zeitliche und nervliche sowie körperliche Belastungen. Neben der Arbeitszufriedenheit werden verschiedene Belastungen bei der eigenen beruflichen Tätigkeit erfasst. In diesem Kapitel werden zwei besonders wichtige Aspekte der Belastungen bei der eigenen Tätigkeit dargestellt: Die Belastungen durch Stress, im Folgenden als zeitliche und nervliche Belastungen bezeichnet („Inwieweit sind Sie belastet durch Stress, das heißt starken Arbeits- oder Termindruck, nervliche Anspannungen?"); sowie Belastungen durch körperliche Aktivitäten („Inwieweit sind Sie belastet durch anstrengende oder einseitige körperliche Aktivitäten, wie zum Beispiel Tragen schwerer Gegenstände, langes Stehen oder Sitzen“). Die Belastungen wurden im DEAS in den Jahren 2002, 2008 und 2014 abgefragt. Für die Analysen wird zwischen stark belasteten (,sehr belastet $\%$,ziemlich belastet') und wenig belasteten Personen (,kaum belastet'/,etwas belastet'/,nicht belastet') unterschieden. Bei der Erfassung von Belastungen wird häufig zwischen einer quantitativen und einer qualitativen Dimension unterschieden (Lohmann-Haislah, 2012). Die quantitativen Belastungen erfassen dabei die Häufigkeit des Auftretens einer belastenden Situation oder Aktivität, wohingegen

2 Die Daten des DEAS können für wissenschaftliche Zwecke kostenlos beim Forschungsdatenzentrum des DZA (www.fdz-dza.de) bezogen werden.

Arbeitszufriedenheit. Die aktuelle Arbeitszufriedenheit wird seit 2002 durch folgende Frage er- 
sich die qualitative Belastung auf die Wahrnehmung der Situation oder Aktivität als belastend bezieht. Aus anderen Studien ist bekannt, dass die quantitative Belastung generell höher ist als die qualitative. Da die Personen im DEAS angeben sollen, wie stark sie diese Anforderungen belasten, zielen die Analysen in diesem Kapitel auf die qualitative Dimension der Belastungen.

Unter- und Überforderung. Nicht nur anstrengende, sondern auch unterfordernde Tätigkeiten können Personen belasten. Daher wird im DEAS seit 2008 erfasst, inwieweit Erwerbstätige ihre eigene Arbeitspassung bewerten („Fühlen Sie sich angesichts Ihrer Qualifikation auf dieser Stelle eher unterfordert, genau richtig gefordert, oder eher überfordert?"). Für die Analysen wird zwischen den Kategorien ,eher unterfordert', ,genau richtig gefordert' und ,eher überfordert' unterschieden.

Erwerbstätigkeit im Ruhestand. Im DEAS wird ebenfalls die Erwerbstätigkeit im Ruhestand erfasst. Es wurde in jeder Befragung von 1996 bis 2014 abgefragt, ob eine Person im Ruhestand erwerbstätig ist. Daneben liegen für das Jahr 2014 noch Informationen zur derzeitigen beruflichen Stellung vor (,als Arbeiter/in oder Angestellte/r beschäftigt' ${ }^{\prime}$ oder , selbstständig erwerbstätig'), ob es sich um den gleichen Arbeitgeber wie vor dem Ruhestand handelt, und wie der Umfang der beruflichen Tätigkeit ist (Stunden pro Woche).

Gruppierungsvariablen. Altersunterschiede wurden anhand von Sechs-Jahres-Altersgruppen untersucht. Dadurch konnten Überschneidungen zwischen Erhebungszeitpunkten und Altersgruppen vermieden werden ( 42 bis 47 Jahre, 48 bis 53 Jahre, 54 bis 59 Jahre, 60 bis 65 Jahre, 66 bis 71 Jahre, 72 bis 77 Jahre, 78 bis 83 Jahre). Personen, die beispielweise 200854 bis 59 Jahre alt waren, sind 2014 zwischen 60 bis 65 Jahre alt. Weiterhin wurden Geschlechtsunterschiede und Regionsunterschiede (Ost-/Westdeutschland) untersucht. Zur Untersuchung von Bildungsunterschieden wurden - basierend auf einer reduzierten ISCED-Klassifizierung - drei Gruppen betrachtet: Personen mit niedrigem, mittlerem und hohem Bildungsabschluss (vgl. Kapitel 2).

Analysen. In den Abbildungen und im Text sind gewichtete (gruppenspezifische) Mittelwerte oder prozentuale Verteilungen angegeben. Um Gruppenunterschiede auf Signifikanz zu testen, wurden logistische oder multinomiale Regressionen berechnet, wobei für die Stratifizierungsvariablen Altersgruppe, Geschlecht und Region (Ost-/Westdeutschland) kontrolliert wurde. Das genaue Vorgehen ist in Kapitel 2 beschrieben.

\subsection{Wandel der Erwerbsbeteiligung älterer Erwerbspersonen zwischen 1996 und 2014}

Im folgenden Abschnitt wird die Veränderung der Erwerbsbeteiligung in der zweiten Lebenshälfte zwischen 1996 und 2014 betrachtet. Zunächst soll aber die Frage beantwortet werden, wer im Jahr 2014 erwerbstätig ist. Abbildung 3-1 zeigt, dass im Jahr 2014 74,1 Prozent der 40- bis 65-Jährigen erwerbstätig sind. 13,8 Prozent befinden sich bereits im Altersrentenbezug vor dem gesetzlichen Ruhestandsalter und weitere 12,2 Prozent gehören zu der Gruppe der Nicht-Erwerbstätigen.

Für das Jahr 2014 gibt es signifikante Unterschiede in der Erwerbsbeteiligung nach Alters- gruppen. Die Anteile der Erwerbstätigen liegen bei den 42 - bis 47 -Jährigen sowie den 48 - bis 53-Jährigen über dem Durchschnitt (86,3 Prozent beziehungsweise 87,2 Prozent) und unterscheiden sich nicht signifikant zwischen diesen beiden Altersgruppen (Abbildung 3-2). Der Anteil der erwerbstätigen Personen im Alter von 54 bis 59 Jahren liegt bei 76,1 Prozent und damit signifikant unter dem der 42 - bis 47 -Jährigen sowie der 48- bis 53-Jährigen. In der Altersgruppe der 60- bis 65-Jährigen ist der Anteil der Erwerbstätigen mit 38,8 Prozent am geringsten. 


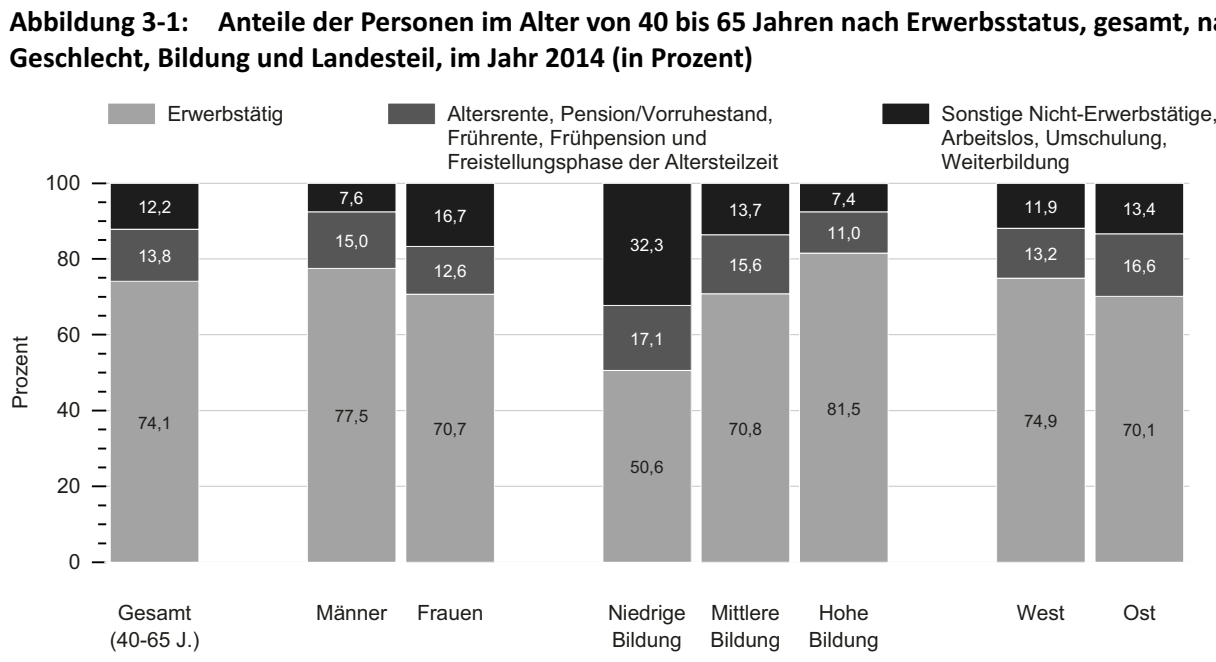

Quelle: DEAS 2014 ( $n=3.531)$; gewichtet, gerundete Angaben; $(p<, 05)$.

Signifikante Unterschiede für alle Kategorien zwischen Männern und Frauen, zwischen allen Bildungsgruppen sowie zwischen den Landesteilen.

Die Erwerbsbeteiligung unterscheidet sich im Jahr 2014 bei den 40 - bis 65-Jährigen weiterhin nach Geschlecht, Bildung und Region. In Abbildung 3-1 sind die Anteile für verschiedene Gruppen im Jahr 2014 dargestellt. Wie vermutet, sind Männer mit 77,5 Prozent eher erwerbstätig als Frauen mit 70,7 Prozent. Für Bildung zeigt sich, dass im Jahr 2014 50,6 Prozent der Personen mit niedrigem Bildungsniveau erwerbstätig sind, jedoch 70,8 Prozent der Personen mit mittlerem Bildungsniveau und 81,5 Prozent der Personen mit hohem Bildungsniveau. Auch zwischen den Landesteilen unterscheidet sich die Erwerbsquote. Im Jahr 2014 sind 74,9 Prozent der Westdeutschen erwerbstätig, während es bei den Ostdeutschen 70,1 Prozent sind.

\section{Im Jahr 2014 sind mehr Menschen in der Lebensphase vor dem Ruhestand erwerbstätig als 1996.}

In Abbildung 3-2 ist zu sehen, dass sich das Niveau der Erwerbsbeteiligung über die Zeit erhöht hat. Wie erwartet, ist der Anteil der erwerbstätigen Personen an der Bevölkerung im Alter von 40 bis 65 Jahren seit 1996 von 60,2 Prozent auf 74,1 Prozent im Jahr 2014 gestiegen.
Im Gegensatz dazu ist der Anteil der Personen mit Rentenbezug von 17,2 Prozent im Jahr 1996 auf 13,8 Prozent im Jahr 2014 und der Anteil der Nicht-Erwerbstätigen (unter anderem Arbeitslosigkeit, Weiterbildung, Hausfrauen/-männer) an der Bevölkerung im Alter von 40 bis 65 Jahren von 22,7 Prozent im Jahr 1996 auf 12,2 Prozent im Jahr 2014 geschrumpft.

Die Erwerbsbeteiligung unterscheidet sich auch zwischen den Altersgruppen. $\mathrm{Zu}$ allen Befragungszeitpunkten ist $\mathrm{zu}$ erkennen, dass der Anteil von Personen, die erwerbstätig sind, mit höherem Alter geringer ist. Weiterhin zeigt sich, dass über die Befragungszeitpunkte die Erwerbsbeteiligung in allen Altersgruppen der 40- bis 65-Jährigen zugenommen hat. Zudem verläuft diese Entwicklung über die Zeit in den Altersgruppen unterschiedlich. In den Altersgruppen der 42- bis 47-Jährigen sowie der 48- bis 53-Jährigen hat sich der Anteil der Erwerbstätigen von 1996 zu 2014 um 7,4 beziehungsweise 9,6 Prozentpunkte erhöht, sodass der Anteil der Erwerbstätigen an der Bevölkerung 2014 für beide Altersgruppen bei 86,3 beziehungsweise 87,2 Prozent liegt. Dagegen hat sich bei den 54 - bis 59-Jährigen und den 60- bis 65-Jährigen im gleichen Zeitraum der Anteil der Erwerbstätigen um knapp 20 Prozentpunkte erhöht. In der ältesten 
Altersgruppe ist der Anteil von $1996 \mathrm{zu} 2002 \mathrm{zu}-$ nächst nicht angestiegen. Von 2002 zu 2014 hat sich anschließend der Anteil der Erwerbstätigen im Alter von 60 bis 65 Jahren von 17,6 Prozent auf 38,8 Prozent verdoppelt. Insgesamt sind die An- teile der älteren Erwerbstätigen seit 1996 gestiegen, die Erwerbsbeteiligung der 54- bis 59-Jährigen und der 60- bis 65-Jährigen ist im Jahr 2014 weiterhin signifikant niedriger als die der 42 - bis 47-Jährigen und der 48- bis 53-Jährigen.

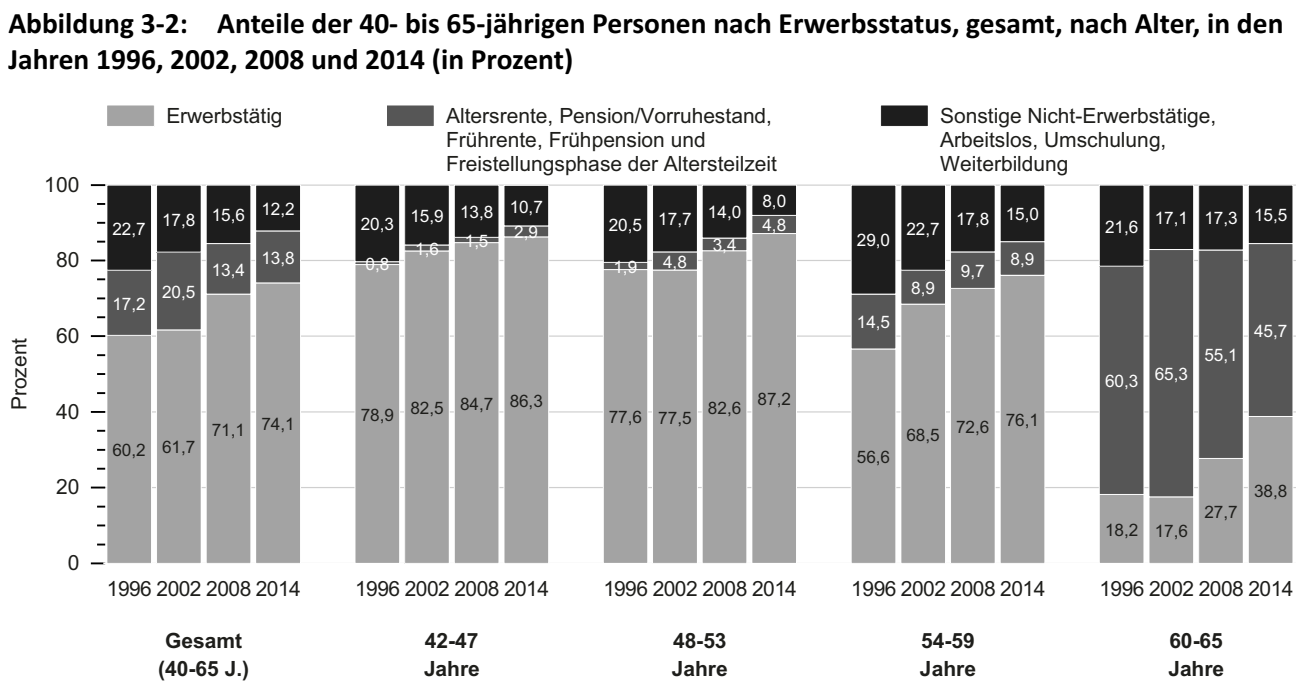

$\overline{\text { Quelle: DEAS } 1996}(n=3.134), 2002(n=1.844), 2008(n=3.572), 2014(n=3.531)$; gewichtet, gerundete Angaben; $(p<, 05)$.

Signifikante Zunahme der Kategorie ,Erwerbstätig' im Alter 40 bis 65 Jahre zwischen 1996 und 2014. Signifikante Abnahme der Kategorie ,Altersrente' sowie ,sonstige Nicht-Erwerbstätige' zwischen 1996 und 2014. In allen Altersgruppen steigt der Anteil der Erwerbstätigen von 1996 bis 2014 an. Signifikante Zunahme des Anteils der Altersrentnerinnen und Altersrentner bei den 42- bis 53-Jährigen, aber signifikante Abnahme bei den 54- bis 65-Jährigen. In allen Altersgruppen (außer bei den 60- bis 65-Jährigen) sinkt der Anteil der sonstigen Nicht-Erwerbstätigen von 1996 bis 2014.

Unterschiede zeigen sich ebenfalls bei der Entwicklung der Anteile der Personen mit Altersrentenbezug vor dem gesetzlichen Ruhestandsalter. Während in den beiden jüngeren Altersgruppen (42- bis 47-Jährigen und 48- bis 53-Jährigen) die Anteile der Personen mit Altersrentenbezug im Gesamttrend geringfügig angestiegen sind, ist deren Anteil bei den 54- bis 65-Jährigen signifikant gesunken: Die Anteile der 54- bis 59-Jährigen mit Altersrentenbezug betrugen 1996 noch 14,5 Prozent. Im Jahr 2014 sind es nur noch 8,9 Prozent. Auch bei den 60- bis 65-Jährigen waren es im Jahr 1996 60,3 Prozent und im Jahr 2014 lediglich 45,7 Prozent. Der gleiche Trend zeigt sich für sonstige Nicht-Erwerbstätige. Ihr Anteil an den Erwerbspersonen hat im Gesamttrend abgenommen (zur Veränderung der Renteneintrittsmuster über die Zeit (vgl. Kapitel 4)).
Im Jahr 2014 bestehen weiterhin signifikante Unterschiede in den Anteilen der Erwerbsbeteiligung zwischen Männern und Frauen. Trotz des immer noch bestehenden Unterschiedes, haben sich die Anteile zwischen Männern und Frauen jedoch seit 1996 Schritt für Schritt einander angeglichen (vgl. Tabelle A 3-1 im Anhang). Das zeigt, dass die schrittweise Erhöhung des gesetzlichen Renteneintrittsalters gleichermaßen auf den Zeitpunkt des Ruhestandsüberganges von Männern als auch von Frauen wirkt. Die Differenzen in der Erwerbsbeteiligung zwischen den Bildungsgruppen sind über die Zeit stabil geblieben (ohne Abbildung). Der Ost- und Westunterschied in den Anteilen der Erwerbsbeteiligung besteht konstant seit 1996 (vgl. Tabelle A 3-1 im Anhang). 


\subsection{Bewertung und Wandel der eigenen Arbeit von Erwerbstätigen}

Angesichts der steigenden Erwerbsbeteiligung vor allem der älteren Erwerbstätigen, wird im Folgenden untersucht, wie sich die Bewertung der Arbeitssituation für Erwerbstätige in der zweiten Lebenshälfte verändert hat. Dabei wird auf die Arbeitszufriedenheit, die Qualität der Belastungen sowie die subjektiv wahrgenommene Passung der Leistungsfähigkeit der Erwerbstätigen mit ihrer Arbeit eingegangen.

Die berichtete allgemeine Arbeitszufriedenheit ist sehr hoch. Im Jahr 2014 berichten 85,2 Prozent der Erwerbstätigen im Alter von 40 bis 65 Jahren, dass sie insgesamt eher oder sehr zufrieden mit ihrer Arbeit sind (vgl. Tabelle A 3-2 im Anhang). Ein Vergleich zwischen den Altersgruppen zeigt für das Jahr 2014 keinen Unterschied bei der berichteten Arbeitszufriedenheit. Der Anteil der zufriedenen Erwerbstätigen ist in allen Altersgruppen gleich hoch. Ebenso gibt es 2014 weder signifikante Unterschiede zwischen Männern und Frauen noch zwischen Bildungsgruppen.

Die Arbeitszufriedenheit hat sich seit 2002 nicht verändert (vgl. Tabelle A 3-2 im Anhang). Das ist bemerkenswert, denn obwohl Personen die Austrittswege aus der Erwerbsarbeit erschwert wurden und damit insgesamt mehr Personen erwerbstätig sind, sind diese nicht unzufriedener. Der hohe Anteil der Arbeitszufriedenheit hat sich seit 1996 auch in keiner der Altersgruppen, zwischen Männern und Frauen oder den Bildungsgruppen signifikant verändert. Diese Befunde passen zu der Argu- mentation, dass die Arbeitszufriedenheit einer Person im Lebenslauf relativ konstant ist, wobei sich Personen in ihrer Arbeitszufriedenheit vor allem durch psychologische Merkmale und nicht durch sozio-ökonomische Merkmale unterscheiden.

Der DEAS erfasst neben der Zufriedenheit mit der eigenen beruflichen Tätigkeit auch die qualitative Dimension von Arbeitsbelastungen, welche auf die Einschätzung abzielt, ob die Anforderungen der Arbeit für die Person belastend sind. Im Folgenden wird zuerst auf die zeitlichen und nervlichen Belastungen eingegangen und danach auf die Belastungen durch körperliche Aktivitäten. Im Jahr 2014 liegt der Anteil der Erwerbstätigen, die sich zeitlich und nervlich belastet fühlen, bei 47,4 Prozent (Abbildung 3-3a).

Im Jahr 2014 gibt es zwar keinen linearen Trend in den Altersunterschieden (Abbildung 3-3a), aber die zeitlichen und nervlichen Belastungen unterscheiden sich dennoch zwischen den einzelnen Altersgruppen. Der Anteil der 42- bis 47-Jährigen, die sich zeitlich und nervlich belastet fühlen, liegt bei 44,7 Prozent. Von dieser Gruppe unterscheiden sich die 48bis 53-Jährigen nicht, jedoch fühlt sich ein signifikant größerer Anteil der 54- bis 59-Jährigen mit 51,4 Prozent zeitlich und nervlich belastet. Bei den 60- bis 65-Jährigen liegt der Anteil der Personen mit zeitlichen und nervlichen Belastungen mit 43,9 Prozent wieder niedriger als bei den 54 - bis 59-Jährigen und sogar den 42 - bis 47-Jährigen. 
Abbildung 3-3: Anteile der ziemlich oder sehr belasteten Erwerbstätigen (zeitliche und nervliche Belastungen, körperliche Belastungen), gesamt, nach Alter, Geschlecht und Bildung, im Jahr 2014 (in Prozent)

\section{a) Zeitliche und nervliche Belastungen}

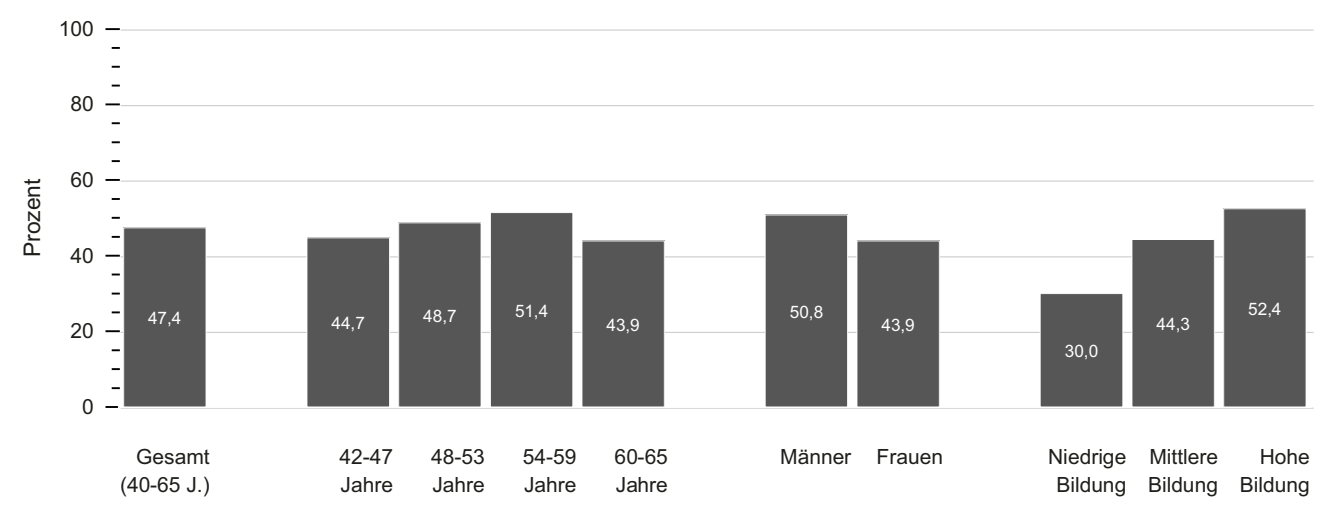

\section{b) Körperliche Belastungen}

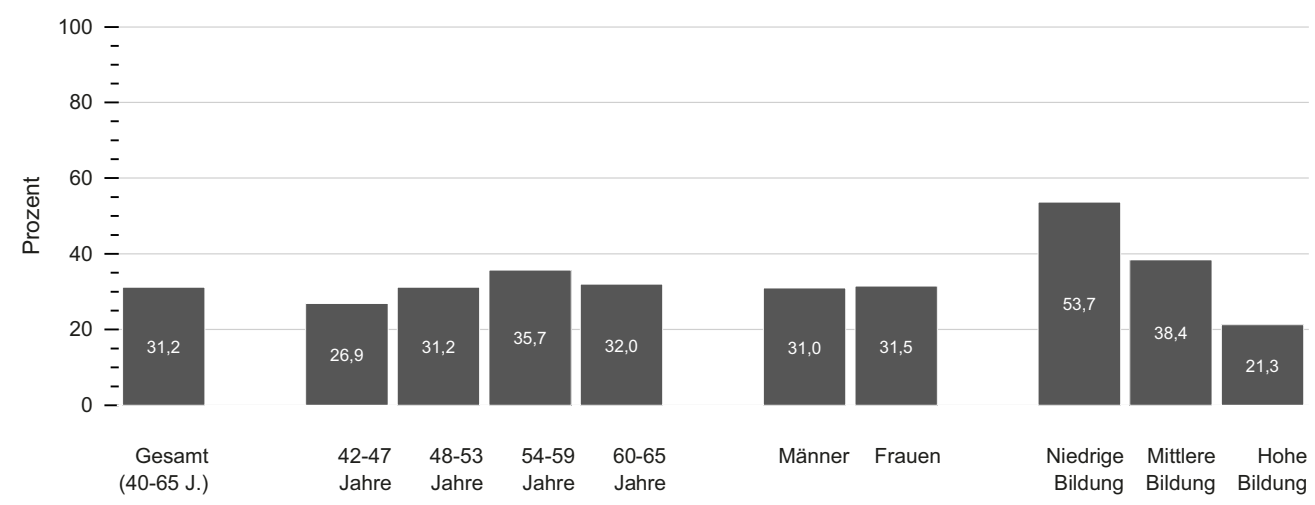

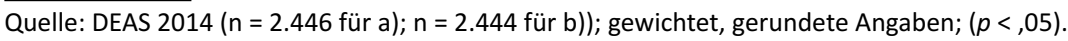

a) Signifikante Altersunterschiede nur zwischen 42- bis 47-Jährigen und 54- bis 59-Jährigen. Signifikante Geschlechts- und Bildungsunterschiede. b) Signifikante Unterschiede nur zwischen 54- bis 59-Jährigen und 42- bis 47-Jährigen sowie 60- bis 65-Jährigen. Keine signifikanten Geschlechtsunterschiede. Signifikante Unterschiede zwischen allen Bildungsgruppen.

\section{Belastungen im Rahmen der Erwerbstätigkeit unterscheiden sich zwischen den Bildungsgruppen.}

Es zeigen sich außerdem Geschlechtsunterschiede: Männer fühlen sich eher zeitlich und nervlich belastet (50,8 Prozent) als Frauen (43,9 Prozent; Abbildung 3-3a). Deutliche Unterschiede zeigen sich im Jahr 2014 auch nach dem Bildungsniveau. Wie erwartet, sind die Anteile der zeitlich und nervlich belasteten Personen bei den Personen mit hohem Bildungsniveau mit 52,4 Prozent höher als bei Personen mit mittlerem und niedrigem Bildungsniveau (44,3 Prozent beziehungsweise 30,0 Prozent).

Seit 2008 ist der Anteil der berichteten zeitlichen und nervlichen Belastungen von 44,0 Prozent auf 47,4 Prozent gestiegen (Abbildung 3-4a). Im Zeitvergleich haben sich die Anteile der berichteten zeitlichen und nervlichen Belastungen 
lediglich bei den 48 - bis 53 -Jährigen signifikant erhöht. In dieser Altersgruppe ist eine Zunahme von 40,9 Prozent im Jahr 2002 zu 48,7 Prozent im Jahr 2014 zu verzeichnen. Für das Jahr 2014 sind demnach insgesamt nicht mehr ältere Erwerbstätige zeitlich und nervlich belastet als 2002. Der Anteil der Personen, die von zeitlichen und nervlichen Belastungen berichten, hat sich zwischen Männern und Frauen seit 2002 nicht verändert (vgl. Tabelle A 3-3 im Anhang). Die Unterschiede zwischen den Bildungsgruppen haben sich im Zeitvergleich unterschiedlich verändert. Für Personen mit niedrigem und hohem Bildungsniveau sind die Anteile der zeitlich und nervlich belasteten Personen zwischen 2002 und 2014 stabil geblieben (Abbildung 3-4a). Zwischen 2008 und 2014 hat sich der Anteil der von zeitlichen und nervlichen Belastungen berichtenden Personen mit mittlerem Bildungsniveau von 39,2 Prozent auf 44,3 Prozent erhöht. Die Anteile der Personen, die 2014 von körperlichen Belastungen berichten, ist mit 31,2 Prozent niedriger als die berichteten zeitlichen und nervlichen Belastungen (Abbildung 3-3b). Wie bei zeitlichen und nervlichen Belastungen, zeigt sich im Jahr 2014 kein linearer Verlauf über die Altersgruppen. Die 42- bis 47-Jährigen berichten seltener von körperlichen Belastungen als die 54- bis 59-Jährigen.

Auch wenn sich bezüglich der körperlichen Arbeitsbelastungen keine Unterschiede zwischen Männern und Frauen finden lassen, zeigen sich Unterschiede nach Bildung um so deutlicher (Abbildung 3-3b): Der Anteil der Personen mit hohem Bildungsniveau, welche körperliche Belastungen berichten, ist mit 21,3 Prozent geringer als der von Personen mit einem mittleren (38,4 Prozent) oder niedrigem Bildungsniveau (53,7 Prozent).

\section{Im Jahr 2014 berichten mehr Erwerbstätige von Belastungen als 2002.}

Die Annahme, dass auch die Bewertung der körperlichen Belastungen im historischen Wandel aufgrund der fortschreitenden Technisierung abgenommen habe, kann nicht bestätigt werden (Abbildung 3-4b). Im Gegenteil: Der Anteil von Personen, die körperliche Belastungen berichten, ist von 25,3 Prozent im Jahr 2002 auf 31,2 Prozent im Jahr 2008 deutlich angestiegen, um zwischen 2008 und 2014 das Niveau zu halten. Werden die Veränderungen der berichteten Belastungen über die Befragungszeitpunkte 2002, 2008 und 2014 nach Altersgruppen unterschieden, zeigt sich kein einheitlicher Trend. Auch zwischen Männern und Frauen gibt es keine signifikanten Unterschiede im Zeitvergleich bei den berichteten körperlichen Belastungen (vgl. Tabelle A 3-4 im Anhang).

Die Unterschiede zwischen den Bildungsgruppen variieren im Zeitvergleich. Es ergibt sich jedoch ein anderes Bild als bei den zeitlichen und nervlichen Belastungen. Der Anteil der Personen mit einem niedrigen Bildungsniveau stieg im Gesamttrend bei den berichteten körperlichen Belastungen bei der eigenen Arbeit von 32,4 Prozent im Jahr 2002 auf 53,7 Prozent im Jahr 2014 (Abbildung 3-4b). Der gleiche Trend lässt sich bei Personen mit einem mittleren Bildungsniveau finden. Hier ist der Anteil von 29,8 Prozent im Jahr 2002 auf 38,4 Prozent im Jahr 2014 gestiegen. Bei Personen mit einem hohen Bildungsniveau ist der Anteil der berichteten körperlichen Belastungen nur in dem Zeitraum von 2002 (18,3 Prozent) zu 2008 (24,4 Prozent) gestiegen. Im Gesamtzeitraum von 2002 bis 2014 haben sich die Anteile aber nicht signifikant verändert. 
Abbildung 3-4: Anteile der ziemlich oder sehr belasteten Erwerbstätigen (zeitliche und nervliche Belastungen, körperliche Belastungen), gesamt, nach Alter und Bildung, in den Jahren 1996, 2002, 2008 und 2014 (in Prozent)

a) Zeitliche und nervliche Belastungen

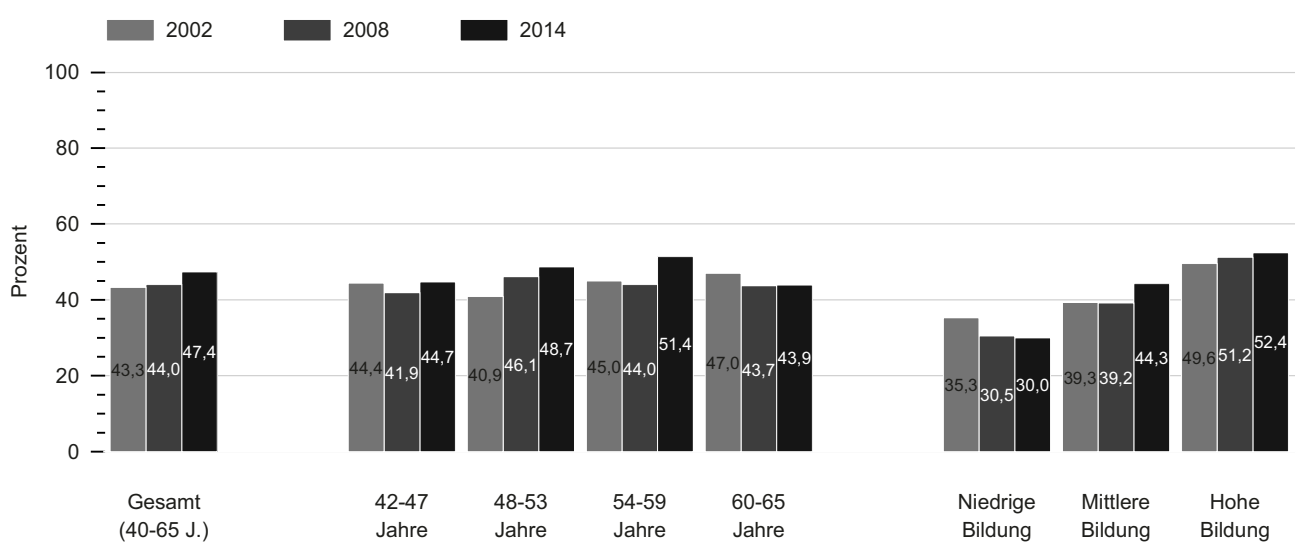

b) Körperliche Belastungen

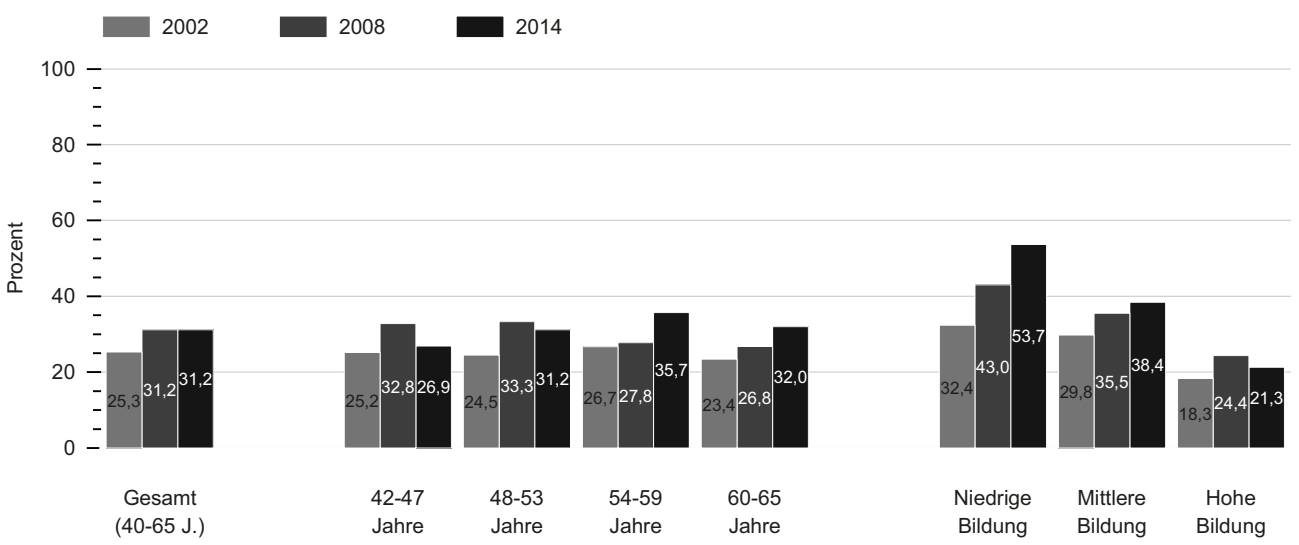

Quelle: DEAS 2002 ( $n=1.098$ für a); $n=1.056$ für b)), $2008 \mathrm{n}=(2.337$ für $\mathrm{a}) ; \mathrm{n}=2.234$ für $\mathrm{b})), 2014(\mathrm{n}=2.466$ für a); $\mathrm{n}=2.444$ für b)); gewichtet, gerundete Angaben; $(p<, 05)$.

a) Stabilität zwischen 2002 und 2008, signifikante Zunahme zwischen 2008 und 2014 für gesamt. Stabilität zwischen 2002 und 2008 in allen Altersgruppen. Zwischen 2008 und 2014 signifikante Zunahme nur bei 54- bis 59-Jährigen. Signifikante Zunahme zwischen 2002 und 2014 nur bei 48- bis 53-Jährigen. Stabilität zwischen 2002 und 2008 in allen Bildungsgruppen. Signifikante Zunahme zwischen 2008 und 2014 nur bei mittlerer Bildung. b) Signifikante Zunahme zwischen 2002 und 2008, Stabilität zwischen 2008 und 2014 für gesamt. Signifikante Zunahme zwischen 2002 und 2008 nur bei 42- bis 53-Jährigen. Zwischen 2008 und 2014 signifikante Unterschiede nur bei 42- bis 47-Jährigen und 54- bis 59-Jährigen. Zwischen 2002 und 2014 signifikante Zunahme bei niedriger und mittlerer Bildung. Bei hoher Bildung signifikante Zunahme zwischen 2002 und 2008 und Stabilität zwischen 2008 und 2014 .

Zusammenfassend zeigt sich, dass seit 2008 zeitliche und nervliche Belastungen und seit 2002 körperliche Belastungen bei den 40- bis 65-Jäh- rigen im allgemeinen Trend zugenommen haben. Auch wenn die Erwerbsquote besonders für höhere Altersgruppen gestiegen ist, zeigt 
sich für diese Personen keine spezifische $\mathrm{Zu}$ nahme der Bewertung ihrer Arbeitsbelastungen über die Befragungszeitpunkte.

\section{Der Großteil der Erwerbstätigen ist im Jahr 2014 zufrieden mit ihrer Arbeit und fühlt sich weder über- noch unterfordert.}

Auch wenn jede zweite erwerbstätige Person im Alter von 40 bis 65 Jahren von zeitlichen und nervlichen Belastungen und jede dritte von körperlichen Belastungen berichtet, fühlt sich im Jahr 2014 ein Großteil der Erwerbstätigen (81,2 Prozent) durch ihre Tätigkeit genau richtig gefordert, 16,1 Prozent fühlen sich eher unterfordert und 2,8 Prozent eher überfordert (Abbildung 3-5). Es zeigen sich keine signifikanten Unterschiede zwischen den verschiedenen Altersgruppen. Die Anforderungspassung unterscheidet sich auch nicht signifikant zwischen Männern und Frauen. Obwohl Männer sich eher durch zeitlich und nervlich belastende Arbeitsbedingungen belastet fühlen, geben sie nicht eher als Frauen an, dass sie sich durch ihre Tätigkeit überfordert fühlen. Weiterhin konnte kein Zusammenhang der Über- oder Unterforderung zwischen den Bildungsgruppen gefunden werden.

Im Vergleich zum Jahr 2008 haben sich die Anteile der über-, unter- oder genau richtig geforderten Personen nicht signifikant verändert (vgl. Tabelle A 3-5 im Anhang). Der Anstieg der zeitlichen und nervlichen sowie körperlichen Belastungen über die Befragungszeitpunkte scheint somit nicht mit einem ansteigenden Anteil an überforderten Personen einherzugehen.

Abbildung 3-5: Anteile der Erwerbspersonen bis 65 Jahre, die berichten sich von ihrer Erwerbsarbeit unter-, über- oder genau richtig gefordert zu fühlen, gesamt, nach Alter, Geschlecht und Bildung, im Jahr 2014 (in Prozent)

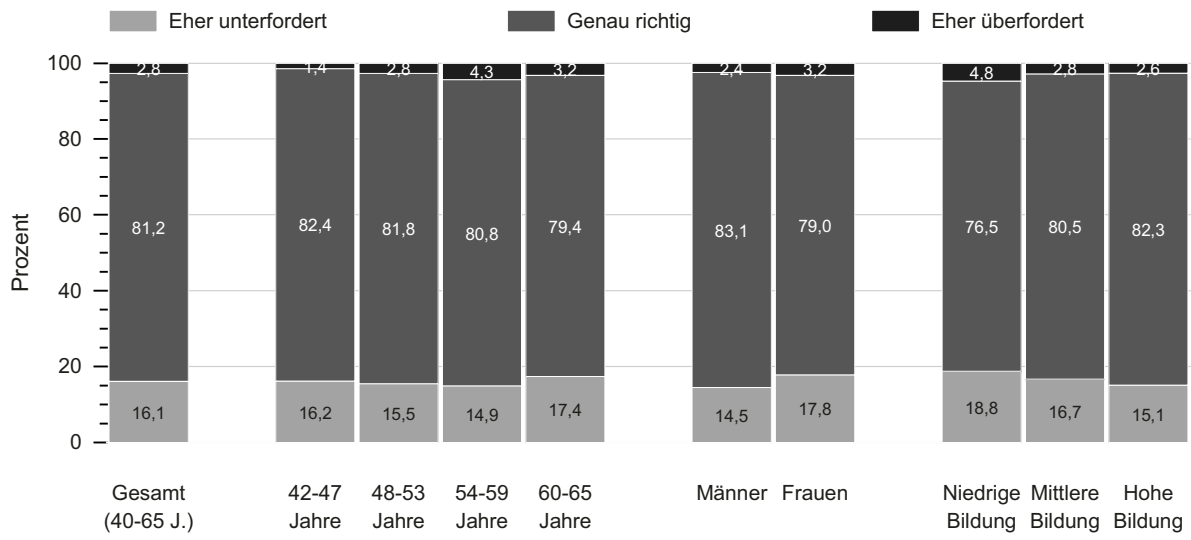

Quelle: DEAS 2008 ( $n=2.385), 2014$ ( $n=2.463)$; gewichtet, gerundete Angaben; $(p<, 05)$.

Keine signifikanten Unterschiede zwischen Altersgruppen, Geschlechtern, sowie Bildungsgruppen. Nur bei der Kategorie ,genau richtig' signifikanter Geschlechtsunterschied.

Zusammenfassend zeigt sich, dass Erwerbstätige in der zweiten Lebenshälfte eine hohe Arbeitszufriedenheit aufweisen und sich trotz zeitlichen und nervlichen Belastungen oder körperlichen Aktivitäten kaum überfordert fühlen. Die Belastungen scheinen über die Altersgruppen erst zuzunehmen, um dann in den letzten Jahren vor dem Ruhestandsüber- gang wieder abzunehmen. Trotzdem zeigt sich, dass sich ältere Erwerbstätige im Alter von 54 bis 65 Jahren nicht von den Erwerbstätigen im mittleren Erwachsenenalter von 42 bis 53 Jahre bezüglich der Arbeitszufriedenheit sowie einer Unter- beziehungsweise Überforderung unterscheiden. 
Bei der Untersuchung der verschiedenen Bildungsgruppen ergibt sich ein anderes Bild. Das Bildungsniveau wirkt sich zwar nicht auf die Arbeitszufriedenheit und die Anforderungspassung aus. Stattdessen zeigen sich in Bezug auf die Belastungen deutliche Unterschiede zwischen Personen mit einem hohen Bildungsniveau und
Personen mit einem niedrigen Bildungsniveau. Während Personen mit niedrigem Bildungsniveau eher von körperlichen Belastungen berichten, sind Personen mit hohem Bildungsniveau eher von zeitlichen und nervlichen Belastungen betroffen.

\subsection{Wandel und Ausgestaltung der Erwerbsbeteiligung im Ruhestand}

Wie im letzten Abschnitt gezeigt werden konnte, bleibt die berichtete Arbeitszufriedenheit bei den 40 - bis 65 -Jährigen konstant auf einem hohen Niveau, sowohl über die Altersgruppen als auch über die letzten Befragungszeitpunkte hinweg. Da ein immer größer werdender Anteil der älteren Menschen vor dem Ruhestand erwerbstätig ist, lässt sich vermuten, dass der Anteil der Personen steigt, die auch im Ruhestand noch erwerbstätig sind. In diesem Abschnitt wird betrachtet, wer im Jahr 2014 im Ruhestand erwerbstätig gewesen ist und ob sich der Trend zu einem längeren Arbeiten auch im Ruhestand fortsetzt.

Im Jahr 2014 sind 11,6 Prozent der Personen im Ruhestand erwerbstätig (Abbildung 3-6b). Es gibt aber eindeutige Unterschiede in der Erwerbsbeteiligung im Ruhestand nach Altersgruppen. Die Anteile der im Ruhestand Erwerbstätigen liegen bei den 60 - bis 65 -Jährigen sowie den 66- bis 71-Jährigen bei 18,8 Prozent beziehungsweise 17,2 Prozent und unterscheiden sich nicht signifikant zwischen diesen beiden Altersgruppen (Abbildung 3-6a). Der Anteil der im Ruhestand erwerbstätigen Personen ist bei den 72 - bis 77 -Jährigen signifikant geringer und liegt bei 8,9 Prozent. Bei den 78- bis 83-Jährigen sind lediglich 3,0 Prozent im Ruhestand noch erwerbstätig.
Für das Jahr 2014 zeigen sich außerdem signifikante Unterschiede zwischen Männern und Frauen, West- und Ostdeutschland, sowie nach Bildungsabschluss (Abbildung 3-6a): Während bei den Männern 14,7 Prozent im Ruhestand erwerbstätig sind, sind es bei den Frauen lediglich 8,6 Prozent. Ebenso sind mehr Personen mit hoher Bildung im Ruhestand erwerbstätig als Personen mit einem mittleren und niedrigen Bildungsniveau. Wie bereits vor dem Ruhestand, ist auch nach dem Ruhestandsübergang der Anteil der Erwerbstätigen in Westdeutschland mit 12,2 Prozent höher als der der Ostdeutschen (9,0 Prozent).

\section{Ein zunehmender Anteil an Menschen ist auch nach dem Eintritt in den Ruhestand erwerbstätig.}

Seit 1996 ist der Anteil der erwerbstätigen Personen im Ruhestand kontinuierlich gestiegen. Lag der Anteil der Erwerbstätigen im Ruhestand im Jahr 1996 noch bei 5,1 Prozent, hat sich dieser im Jahr 2014 auf 11,6 Prozent erhöht (Abbildung 3-6b). Am markantesten ist dieser Trend bei den 66- bis 71-Jährigen zu erkennen, bei denen der Anteil von 4,2 Prozent im Jahr 1996 auf 17,2 Prozent im Jahr 2014 gestiegen ist (ohne Abbildung, vgl. Tabelle A-3-6 im Anhang). 


\section{Abbildung 3-6: Anteile der Erwerbstätigen im Ruhestand im Alter von 60 bis 85 Jahren, nach Alter, Geschlecht, Bildung und Landesteil, in den Jahren 1996, 2002, 2008 und 2014 (in Prozent)}

a) Nach Alter, Geschlecht, Bildung und nach Landesteil, 2014

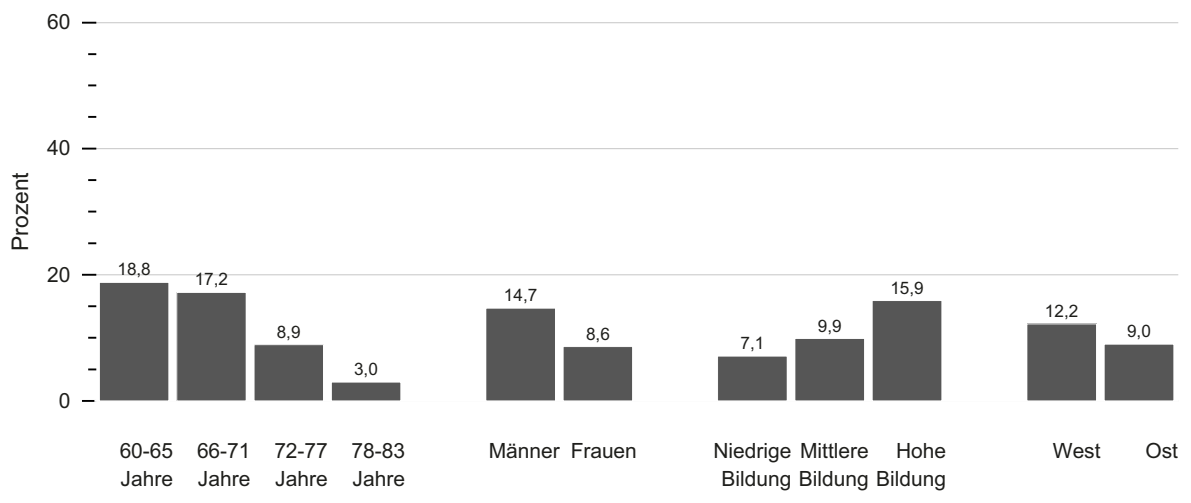

b) Zeitvergleich, 1996, 2002, 2008 und 2014

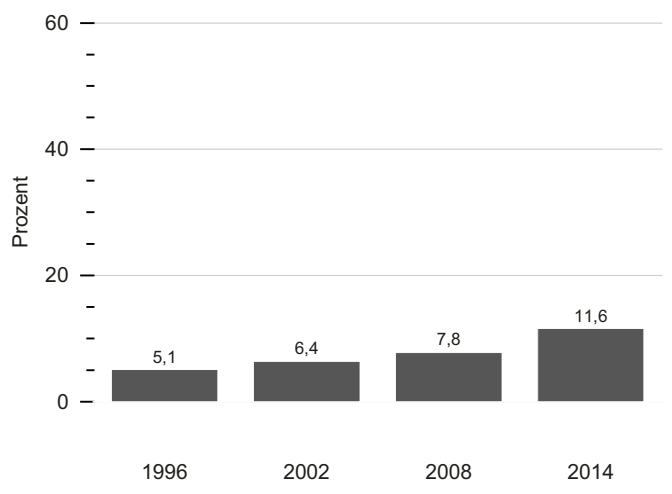

Quelle: DEAS $1996(n=1.845), 2002(n=1.439), 2008(n=2.819), 2014(n=2.711) ;$ gewichtet, gerundete Angaben; $(p<, 05)$.

a) Signifikanter Unterschied zwischen 60- bis 71-Jährigen und Älteren. Signifikante Geschlechtsunterschiede. Signifikanter Unterschied nur zwischen hoher Bildung und mittlerer beziehungsweise niedriger Bildung. Signifikante Regionsunterschiede. b) Signifikante Zunahme zwischen 2008 und 2014, vorher Stabilität.

Im Folgenden wird die Arbeitssituation der Personen betrachtet, die im Ruhestand erwerbstätig sind. 41,5 Prozent der im Ruhestand erwerbstätigen Personen sind 2014 selbstständig tätig, wobei dies stärker für Männer zutrifft (48,2 Prozent) als für Frauen (31,0 Prozent) (ohne Abbildung). Der Anteil der Selbstständigen ist unter den Älteren größer (60- bis 65-Jährige: 24,7 Prozent; 66- bis 71-Jährige: 38,2 Prozent; 72- bis 77-Jährige: 57,3 Prozent beziehungsweise 78- bis 83-Jährige: 66,9 Prozent) (ohne Abbil- dung). Lediglich 26,4 Prozent der im Ruhestand Erwerbstätigen arbeiten bei ihrem früheren Arbeitgeber. Die durchschnittliche Arbeitszeit pro Woche liegt im Jahr 2014 bei 15,5 Stunden (vgl. Tabelle A 3-7 im Anhang). Nur knapp fünf Prozent arbeitet 40 Stunden oder mehr pro Woche. Überraschenderweise unterscheidet sich die Arbeitszeit zwischen den Altersgruppen nicht. Das heißt, dass ältere Personen seltener im Ruhestand erwerbstätig sind, diese aber im Durchschnitt genauso lange arbeiten. Es bleibt unklar, 
ob dies daran liegt, dass Personen unabhängig davon, wie viele Stunden sie zuvor gearbeitet haben, ihre Erwerbstätigkeit im Ruhestand beenden (zufällige Selektion) oder aber, ob Personen die Arbeitszeit mit dem Alter reduzieren und gleichzeitig gerade die Erwerbstätigen ihre Arbeit beenden, die wenige Stunden gearbeitet haben (kompensatorische Selektion). In beiden Fällen bleibt das durchschnittliche Niveau der wöchentlichen Stundenzahl bestehen. Es gibt keinen signifikanten Unterschied in der Arbeitszeit zwischen Männern und Frauen.
In diesem Abschnitt konnte gezeigt werden, dass immer mehr Personen auch im Ruhestand erwerbstätig sind. Gerade in der Altersgruppe der 60- bis 71-Jährigen ist ein starker Anstieg zwischen 1996 und 2014 zu verzeichnen. Die meisten dieser Erwerbstätigen im Ruhestand arbeiten deutlich weniger als 40 Stunden pro Woche. Deutlich wird auch, dass vor allem Personen mit hohem Bildungsniveau im Ruhestand weiter erwerbstätig sind.

\subsection{Diskussion und Implikationen}

Um den strukturellen Veränderungen auf dem Arbeitsmarkt zu begegnen, wurden Wege in die vorzeitige Verrentung geschlossen und das Renteneintrittsalter Schritt für Schritt erhöht. Die Ergebnisse dieses Kapitels zeigen für die Jahre 1996 bis 2014, dass Erwerbstätige länger im Arbeitsmarkt bleiben und dabei gerade der Anteil der Erwerbstätigen im Alter von 54 bis 65 Jahren deutlich angestiegen ist (zu Gestaltung und Wandel der Ruhestandsübergänge vgl. Kapitel 4). Damit decken sich die Ergebnisse aus dem DEAS mit denen der Bundesagentur für Arbeit (2013).

Die meisten Erwerbstätigen im Alter von 40 bis 65 Jahren sind zufrieden oder sehr zufrieden mit ihrer Arbeit. Parallel zu dem gestiegenen Anteil der Erwerbstätigen gibt es aber auch einen etwas größeren Anteil an Personen im Alter von 40 bis 65 Jahren, die von zeitlichen und nervlichen sowie von körperlichen Belastungen bei der Arbeit berichten. Seit 2008 haben die berichteten zeitlichen und nervlichen Belastungen und seit 2002 die berichteten körperlichen Belastungen bei den 40- bis 65-Jährigen im allgemeinen Trend zugenommen. Es ist zu vermuten, dass trotz fortschreitender Technisierung die Belastungen durch einseitige körperliche Aktivitäten gerade durch vermehrtes langes Sitzen gestiegen sind.

Zwar ist die Erwerbsquote vor allem bei den höheren Altersgruppen gestiegen, jedoch zeigt sich für diese Personen keine spezifische $\mathrm{Zu}$ - nahme der Bewertung ihrer Arbeitsbelastungen über die Befragungszeitpunkte. Die Anzahl von Personen, die von Belastungen berichten, scheint über die Altersgruppen erst zuzunehmen, um dann in den letzten Jahren vor dem Ruhestandsübergang wieder abzunehmen. Die von den Arbeitsmarktreformen ausgehenden Schließungsprozesse für den vorzeitigen Ruhestand wirken sich wie bei der Arbeitszufriedenheit nicht auf die Bewertungen der Belastungen aus. Auch wenn es für belastete Erwerbstätige schwerer geworden ist, den Arbeitsmarkt vorzeitig zu verlassen als es noch zu einem früheren Zeitpunkt der Fall war, ist der Anteil der belasteten älteren Erwerbstätigen nicht gestiegen.

Bei der Untersuchung der verschiedenen Bildungsgruppen zeigt sich ein anderes Bild. Das Bildungsniveau wirkt sich zwar nicht auf die Arbeitszufriedenheit und die Anforderungspassung aus. Stattdessen zeigen sich in Bezug auf die Belastungen deutliche Unterschiede zwischen Personen mit einem hohen Bildungsniveau und Personen mit einem niedrigen Bildungsniveau. Während Personen mit niedrigem Bildungsniveau eher von körperlichen Belastungen berichten, sind Personen mit hohem Bildungsniveau eher von zeitlichen und nervlichen Belastungen betroffen.

Neben einem stabilen Anteil an erwerbstätigen Personen, haben sich auch die Anteile der über-, unter- oder genau richtig geforderten 
Personen zwischen 2008 und 2014 nicht verändert. Der Anstieg der psychischen und körperlichen Belastungen scheint demnach nicht mit einer größeren Anzahl an überforderten Personen einherzugehen.

Die größere Anzahl an Personen, die von Belastungen berichtet, könnte zumindest teilweise darauf zurückzuführen sein, dass es für Personen mit hoher Belastung schwieriger geworden ist, vorzeitig aus dem Erwerbsleben auszutreten. Trotzdem bleiben die Arbeitszufriedenheit und die Bewertung der Anforderungspassung auf gleichem, hohem Niveau. Diese Befunde könnten für die Fähigkeit der Erwerbstätigen sprechen mit steigenden körperlichen und psychischen Belastungen umzugehen und sich daran anzupassen. Dies ist für alle Altersgruppen der 40- bis 65-Jährigen in gleichem Maße der Fall. Der Einfluss von nachteiligen Arbeitsbedingungen auf die Erwerbsaustrittswahrscheinlichkeit wird in diesem Kapitel zwar nicht untersucht, jedoch zeigen diese Folgen, weshalb es wichtig und notwendig ist, sich mit diesen Themen zu beschäftigen.

Nicht nur der Anteil der erwerbstätigen Personen vor dem Ruhestand steigt. Auch im Ruhestand sind immer mehr Menschen erwerbstätig. Vor allem der Anteil der im Ruhestand erwerbstätigen 60- bis 71-Jährigen hat zwischen 1996 und 2014 zugenommen, wenn auch mit deutlich weniger als 40 Stunden pro Woche. Erwerbstäti- ge Personen im Ruhestand sind dabei vor allem Männer, Personen mit einem höheren Bildungsniveau und Westdeutsche.

Es ist nicht das Alter, welches ein längeres und zufriedenes Arbeiten bestimmt, sondern die Bildung. Besonders ausgeprägt erscheinen Bildungsunterschiede der 40- bis 65-Jährigen im Bereich von körperlichen und psychischen Belastungen, für die spezifische politische Programme und präventive Maßnahmen gefunden werden könnten. Aus den Ergebnissen lässt sich ableiten, dass älterwerdende Belegschaften vor differenzierten Herausforderungen stehen. Der seit 2002 steigende Anteil von Personen, der von körperlichen oder psychischen Belastungen berichtet, kann in den jeweiligen Bildungsgruppen nicht mehr durch vorzeitige Erwerbsaustritte kompensiert werden, sodass Präventionsmaßnahmen und spezifische Förderung notwendig und sinnvoll erscheinen. Dabei ist jedoch nicht eine allgemeine Reduktion von Anforderungen das Mittel der Wahl, da dies die Gefahr einer Unterforderung birgt. Vielmehr bedarf es flexibler Maßnahmen nah an den Bedürfnissen einzelner Erwerbstätiger. Wie sich an dem seit 1996 kontinuierlich steigenden Anteil von Erwerbstätigen im Ruhestand zeigt, ist ein Teil der Personen in der zweiten Lebenshälfte, unabhängig von ihrem Alter und gesetzlichen Altersgrenzen, willens und fähig, sich aktiv am Arbeitsmarkt einzubringen.

\section{Literatur}

Asef, D., \& Wingerter, C. (2011). Arbeitsmarkt und Erwerbstätigkeit. In: Statistisches Bundesamt \& Wissenschaftszentrum Berlin für Sozialforschung (Hrsg.) Datenreport 2011. Ein Sozialbericht für die Bundesrepublik Deutschland (S. 97-129). Bonn: Bundeszentrale für politische Bildung.

Bäcker, G., Brussig, M., Jansen, A., Knuth, M., \& Nordhause-Janz, J. (2009). Einführung: Beschäftigungsmöglichkeiten für ältere Arbeitnehmer und Arbeitnehmerinnen im Altersübergang. In: G. Bäcker, M., Brussig, A. Jansen, M. Knuth \& J. Nordhause-Janz (Hrsg.) Ältere Arbeitnehmer. Erwerbstätigkeit und soziale Sicherung im Alter (S. 15-45). Wiesbaden: VS Verlag für Sozialwissenschaften.
Bal, A. C., Reiss, A. E. B., Rudolph, C. W., \& Baltes, B. B. (2011). Examining Positive and Negative Perceptions of Older Workers: A Meta-Analysis. The Journals of Gerontology Series B: Psychological Sciences and Social Sciences, 66B(6), 687-698. doi: 10.1093/geronb/ gbr056.

Behrend, C. (2002). Erwerbsarbeit Älterer im Wandel - demographische Herausforderungen und Veränderungen der Arbeitsgesellschaft. In: C. Behrend (Hrsg.) Chancen für die Erwerbsarbeit im Alter. Betriebliche Personalpolitik und ältere Erwerbstätige (S. 11-30). Opladen: Leske + Budrich. 
Bödeker, W., Friedel, H., Friedrichs, M., \& Röttger, C. (2008). The impact of work on morbidity-related early retirement. Journal of public health, 16(2), 97-105.

Brenke, K. (2015). Die große Mehrzahl der Beschäftigten in Deutschland ist mit ihrer Arbeit zufrieden. DIW Wochenbericht, 32/33, 715-722.

Brussig, M. (2010). Anhaltende Ungleichheiten in der Erwerbsbeteiligung Älterer - Zunahme an Teilzeitbeschäftigung. Duisburg/Essen: Institut Arbeit und Qualifikation.

Brussig, M. (2009). Die Erwerbsbeteiligung älterer Arbeitnehmer/-innen in Deutschland im Wandel: Perspektiven der Arbeitsmarktforschung. Zeitschrift für Gerontologie und Geriatrie, 42(4), 281-286.

Bundesagentur für Arbeit (2013). Arbeitsmarktberichterstattung: Der Arbeitsmarkt in Deutschland. Ältere am Arbeitsmarkt. Nürnberg: Bundesagentur für Arbeit.

Büsch, V., Dittrich, D., \& Lieberum, U. (2010). Determinants of Work Motivation and Work Ability among Older Workers and Implications for the Desire for Continued Employment. Comparative Population Studies, 35(4), 931-958.

Engstler, H., \& Romeu Gordo, L. (2014). Arbeiten im Ruhestand - Entwicklung, Faktoren und Motive der Erwerbstätigkeit von Altersrentenbeziehern. In: E. Kistler
\& F. Trischler (Hrsg.) Reformen auf dem Arbeitsmarkt und in der Alterssicherung - Folgen für die Einkunftslage im Alter (S. 115-147). Düsseldorf: Hans-BöcklerStiftung.

Lohmann-Haislah, A. (2012). Stressreport Deutschland 2012. Psychische Anforderungen, Ressourcen und Befinden. Dortmund: Bundesanstalt für Arbeitsschutz und Arbeitsmedizin.

Mümken, S., \& Brussig, M. (2012). Alterserwerbsbeteiligung in Europa. Deutschland im internationalen Vergleich. Duisburg/Essen: Institut Arbeit und Qualifikation.

Ng, T. W. H., \& Feldman, D. C. (2008). The relationship of age to ten dimensions of job performance. Journal of Applied Psychology, 93(2), 392-423. doi: 10.1037/0021-9010.93.2.392.

Rosenstiel, L. von (2009). Unerkannte Potenziale - ältere Beschäftigte aus der Sich der Arbeits- und Organisationspsychologie. In: K. Brauer \& K. Gabriele (Hrsg.) Perspektive 50plus? Theorie und Evaluation der Arbeitsmarktintegration Älterer (S. 41-53). Wiesbaden: VS Verlag für Sozialwissenschaften.

Wurm, S. (2004). Gesundheitliche Potenziale und Grenzen älterer Erwerbspersonen. Expertise im Auftrag der Sachverständigenkommission „Fünfter Altenbericht der Bundesregierung“. Berlin: Deutsches Zentrum für Altersfragen.

Open Access Dieses Kapitel wird unter der Creative Commons Namensnennung 2.5 International Lizenz (http://creativecommons.org/licenses/by/2.5/deed.de) veröffentlicht, welche die Nutzung, Vervielfältigung, Bearbeitung, Verbreitung und Wiedergabe in jeglichem Medium und Format erlaubt, sofern Sie den/die ursprünglichen Autor(en) und die Quelle ordnungsgemäß nennen, einen Link zur Creative Commons Lizenz beifügen und angeben, ob Änderungen vorgenommen wurden.

Die in diesem Kapitel enthaltenen Bilder und sonstiges Drittmaterial unterliegen ebenfalls der genannten Creative Commons Lizenz, sofern sich aus der Abbildungslegende nichts anderes ergibt. Sofern das betreffende Material nicht unter der genannten Creative Commons Lizenz steht und die betreffende Handlung nicht nach gesetzlichen Vorschriften erlaubt ist, ist für die oben aufgeführten Weiterverwendungen des Materials die Einwilligung des jeweiligen Rechteinhabers einzuholen. 